\title{
Diversidad de la enseñanza universitaria y de la práctica del diseño en México
}

Diversity of University Teaching and Design Practice in Mexico

\author{
Volumen 21, Número 2 \\ Mayo - Agosto \\ pp. 1-23
}

\author{
Arodi Morales Holguín \\ Edgar Oswaldo González Bello
}

Citar este documento según modelo APA

Morales Holguín, Arodi., y González Bello, Edgar Oswaldo. (2021). Diversidad de la enseñanza universitaria y de la práctica del diseño en México. Revista Actualidades Investigativas en Educación, 21(2), 1-23. Doi. 10.15517/aie.v21i2.46779 


\title{
Diversidad de la enseñanza universitaria y de la práctica del diseño en México

\author{
Diversity of University Teaching and Design Practice in Mexico
}

\author{
Arodi Morales Holguín ${ }^{1}$ \\ Edgar Oswaldo González Bello²
}

\begin{abstract}
Resumen: La formación universitaria en el diseño demanda el manejo apropiado de métodos para diseñar como proceso de creación, elemento preponderante para la enseñanza y el profesorado. El objetivo del presente artículo es identificar diferencias en el uso de métodos de diseño que se utilizan en la enseñanza y la práctica profesional, según la edad del profesorado. Es un estudio cuantitativo apoyado en el método descriptivo y en la aplicación, en el período 2018-2019, de un cuestionario a 126 personas docentes de México de las áreas de diseño, diferenciadas según su edad. Los resultados revelan una diversidad marcada con la edad: el profesorado de hasta 40 años, quienes posiblemente recibieron una formación envuelta por la tecnología e Internet, conciben el diseño de forma más tradicional, prefiriendo métodos de perfil lineal-secuencial. Desde otro extremo, aquellas personas con edad superior a 40 años, quienes se desarrollaron en un entorno tecnológico con limitantes, evidenciaron perspectivas más amplias y progresistas desde el método sistémico. Esto resultados también mostraron diferencias estadísticamente significativas con respecto a la edad. Se concluye que, en referencia a la enseñanza y a la práctica profesional, el profesorado de menor edad, que probablemente apueste por el dominio tecnológico-digital, se decanta por métodos menos actualizados que privilegian el aspecto técnico; mientras que las personas de mayor edad se aventuran por aquellos de avanzada, de una visión más integral y analítica. Asumir esta situación contribuye a la planificación de estrategias que orienten al mejoramiento de la enseñanza, a la formación en áreas de diseño.
\end{abstract}

Palabras clave: diseño, método, formación profesional, trabajos prácticos.

\begin{abstract}
University training in the field of design demands the appropriate handling of design methods as a process for creation, a preponderant element for teaching and teachers. In this sense, this text identifies differences in the use of design methods that are used in teaching and professional practice according to the age of the teaching staff. It is a quantitative study supported by the descriptive method and the application, in the period 2018-2019, of a questionnaire to 126 teachers from Mexico of the design areas, differentiated according to their age. The results reveal a diversity marked with age: teachers up to 40 years of age, who possibly received training involving technology and the Internet, conceive of design in a more traditional way, preferring linear-sequential profile methods. From another extreme, those over 40 years of age, who are assumed to have been trained in a technological environment with limitations, showed broader and more progressive perspectives from the complexity promoted by the systemic method. These results also showed statistically significant differences with respect to age. It is concluded that, in reference to teaching and professional practice, the younger teachers, who most likely bet on the technological-digital domain, opted for less updated design methods that privilege the technical aspect, while the subjects with greater age adventure by those of advanced, of a more integral, analytical and critical vision. Assuming this situation contributes to the planning of strategies that guide the improvement of teaching and training in the areas of design.
\end{abstract}

Key words: design, method, vocational training, professional practice.

\footnotetext{
${ }_{1}$ Profesor-Investigador de Tiempo Completo de la Universidad de Sonora, Sonora, México. Doctor en Arquitectura Diseño y Urbanismo por la Universidad Autónoma del Estado de Morelos, México. Dirección electrónica: redeshmo@gmail.com ORCID: https://orcid.org/0000-0001-9241-032X

2 Profesor-Investigador de Tiempo Completo de la Universidad de Sonora, Sonora, México. Doctor en Ciencias Sociales por la Universidad de Sonora, México. Dirección electrónica: edgar.gonzalezb@gmail.com ORCID: https://orcid.org/0000-0001-6297-2516
}

Artículo recibido: 18 de noviembre, 2020

Enviado a corrección: 26 de marzo, 2021

Aprobado: 19 de abril, 2021

Los contenidos de este artículo están bajo una licencia Creative Commons 


\section{Introducción}

La enseñanza del diseño en México es un área que ha experimentado un crecimiento notable debido a la oferta universitaria, también por el impacto que el diseño tiene en todos los intersticios del quehacer humano, cuyo propósito, subraya Simon (1996, pp. xii), es aportar objetos o artefactos que actúen como solución en distintos problemas de la sociedad. Este proceso permite, a través de un diálogo social entre personas involucradas, llegar a resultados provechosos (Cramer, Christensen y Ahmed, 2019, p.2). Lo anterior ha hecho que el diseño se convierta en un área de estudio cada vez más trascendental, donde el método empleado para el desarrollo de los productos o diseños destaca como variable preponderante y factor de influencia en cuanto al resultado alcanzado.

No obstante, tanto la formación como el ejercicio profesional del diseño en México parecen darle poca importancia a dicha variable, pese a que el método es de los aspectos preponderantes que determinan la calidad del diseño. Como acentúa Dorochesi (2010, p.96), los métodos y su puesta en práctica repercuten en los productos resultantes. En este sentido, la presente investigación tiene como objetivo identificar el uso de los métodos de diseño en la enseñanza y la práctica profesional en México, de acuerdo con la edad del profesorado, pues se identifican diferencias culturales entre quienes fueron formados antes y después de la era digital.

Respecto a los antecedentes de la investigación, en cuanto a la formación del diseño en México y otras partes del mundo, según Lupton y Miller (2002, p.8), ha sido guiada por el modelo Bauhaus que, a un siglo de distancia, sigue vigente en la formación universitaria en las distintas ramas del diseño: arquitectónico, industrial, gráfico, interiores, modas y otros; presente directamente en los métodos empleados para diseñar. Por método se hace referencia al procedimiento específico para abordar un problema. Para la disciplina del diseño, según Nacif (2015), son:

(...) Todos y cada uno de los procedimientos, técnicas, herramientas, instrumentos que ayudan a lograr un diseño determinado y a su vez, son las distintas clases de actividades que el diseñador utiliza y combina entre sí, dentro de un proceso general de diseño. (p.2)

Desde la perspectiva de Moreno y Rogel (2012, p.97), los métodos utilizados en México para diseñar son variados: van desde intuitivos, responsables con el entorno ambiental y la sociedad, hasta aquellos que están enfocados en la experiencia de las personas. Al respecto, Bertola (2004, p.35) explica que en dicho país, al ser el diseño producto de la Escuela de Artes 
y Oficios, su producción se realiza desde una perspectiva más inclinada a la intuición, la habilidad individual y la experiencia, las cuales han ido impregnándose en la enseñanza y el ejercicio profesional.

Por su parte, el método proyectual de inspiración cartesiana, basado en procesos cognitivos deductivos y que siguen una estructura lineal, ha sido desde el siglo XX la base de los métodos de diseño en un contexto más global. Método considerado como insuficiente dadas las condiciones del entorno y la complejidad de la realidad (Costa, 2009, p.10), donde el racionalismo cartesiano ha ido perdiendo capacidad por lo que surge la necesidad, para el ejercicio del diseño, de una proyección de rutas distintas, más abiertas e incluyentes. Esto paralelamente conduce a cuestionar la enseñanza, los contenidos curriculares y la formación de personas diseñadoras en las universidades mexicanas, donde los métodos de diseño corresponden a la calidad del proceso educativo.

A pesar de la evolución que manifiesta nuestra sociedad red (Castells, 2009, p.24) y su colectividad interconectada, sumergida en entornos tecnológicos, muchos de los métodos de diseño que se siguen aplicando son aquellos surgidos años atrás, donde la enseñanza se ha centrado en los métodos lineales basados en la solución de problemas de otras disciplinas. Mientras tanto, se pudiera esperar que las personas diseñadoras del siglo XXI, al estar ubicados en un entorno de trabajo dominado por tecnologías digitales y rodeados de un vasto volumen visual de conceptos, creaciones, así como información, optarían por un enfoque metodológico de avanzada. No obstante, se percibe una preconcepción de que la acción de diseñar puede ser fácil, bastando crear a través del uso del software y apegándose a diferentes estilos visuales disponibles en Internet, a veces sin atención en el método.

La derivación de esta asimilación hacia la enseñanza del diseño permite suponer una diferenciación marcada en profesorado: por un lado, quienes fueron formados en la etapa previa al dominio de las tecnologías digitales e Internet, hasta mediados de la década de los noventa, que se propone reconocer como etapa predigital; por otra parte, sujetos que fueron formados en la etapa digital, disgregación que igualmente podría tener repercusiones en la formación universitaria de profesionistas del diseño.

Para Garone Gravier (2011, p. 108), en los años noventa el diseño en México comenzaría a trabajarse en un ambiente casi exclusivamente digital, donde la computadora fue un elemento de quiebre generacional, que da inicio a una lucha entre "analógicos" y "digitales". 
Esto produciría una separación ideológica entre personas diseñadoras que fueron formadas en ambientes digital y análogo, evidenciado de mejor forma a partir de Internet, donde lo local y lo global se desvanecen, dando paso a la interacción cultural y comercial global de forma nunca vista. No obstante, dicho acceso tecnológico resultó, según Garone Gravier (2011, p.119), en su talón de Aquiles, dada la veloz caducidad de los saberes técnicos que esto traería; además de la disolución de los trabajos de planta, propios de la etapa predigital, a cambio del incremento de freelancers y minidespachos unipersonales en un período más caracterizado por lo digital.

De acuerdo con esta diferenciación en ambos grupos, el primero (predigitales) al haber sido formado con base en procesos convencionales, más manuales y menos tecnológicos, pudiera asumirse que mostrarían desventajas en comparación con la inercia vivida por el segundo grupo (digitales), generación donde los procesos son claramente dominados por la tecnología digital y donde la información está al alcance de un click. Lo anterior supondría diferencias en su enseñanza y en la práctica del diseño entre uno y otro grupo.

Para asemejar esta diferenciación, se concibe que durante los años sesenta la fotografía se destacaría como un avance importante a favor del diseño (Meggs, 2010, p.493). En cambio, para los noventa la computadora rompería todos los paradigmas con un impacto verdadero en México (Garone Gravier, 2011, p.98); así también en la práctica. Esto promovió una ruptura que marcaría una división generacional entre quienes se desenvolvían en estos años o antes, caracterizados por un perfil más hacia lo creativo, frente a quienes emergieron en el período digital y que apostaban por una cultura visual más universal, y nuevos enfoques apoyados en las tecnologías e Internet.

Con base en lo señalado, el presente artículo busca responder a las siguientes preguntas: en cuánto a los métodos de diseño, ¿qué modelos rigen entre el profesorado de las áreas de diseño?, ¿es la edad y las diferencias generacionales mencionadas entre ambos grupos, un factor de influencia que se relaciona con el uso de los métodos de diseño en la enseñanza y en la práctica? En busca de responder a dichos cuestionamientos, se llevó a cabo una investigación de alcance nacional (México) durante el año 2019, donde se recopiló información del profesorado adscrito a diferentes áreas de diseño tanto en universidades públicas como privadas distribuidas geográficamente en el país. 


\section{Referente teórico}

\subsection{Acerca de los métodos de diseño}

Desde la diferenciación propuesta por Rodríguez Mendoza (2016, p.260) en los enfoques metodológicos utilizados en el mundo natural (objetividad y racionalidad a través de la experimentación controlada) y las humanidades (experiencia humana, donde destaca la metáfora, subjetividad e imaginación), el diseño tendría un modo distinto de aproximación al conocimiento. Dado que su objeto de estudio es sui generis; consecuentemente, sus métodos de investigación, de enseñanza y la disciplina en sí, también lo son.

Al hablar en concreto de métodos en el diseño, es pertinente diferenciar a estos de la metodología. Los primeros exploran el procedimiento a partir de aquellos pasos que se siguen y las reglas que lo rigen, mientras la metodología estudia los métodos. Se identifican dos visiones que definen los métodos de diseño: una es la estructura lineal-secuencial de naturaleza causal, de raíz cartesiana; y, otra son los sistemas complejos, emergidos a mediados del siglo XX, su fundamento se atribuye a Bertalanffy (1951).

La linealidad sería catapultada por Descartes, convirtiéndose en la base del pensamiento científico desde el siglo XVII, paradigma que propone básicamente tomar el fenómeno y dividirlo en partes menores para luego analizar cada una por separado, considerando solo los aspectos principales de cada parte, para luego sumar los resultados o conjeturas de cada una de estas obteniéndose así un resultado final: el conocimiento (Morin, 2007, p.100). Con ello, Descartes postularía como principio de verdad solo aquellas ideas claras y distintas; en otras palabras, aquellas estructuradas desde un orden causal, constante, de naturaleza controlable, fácil de medir y alejado de la dinámica e inestabilidad. De esta forma, condicionó los fenómenos y los redujo a una ecuación simple.

Un esquema de vías causales es aquel que permite dilucidar al fenómeno como organización secuencial similar a una cadena, donde para llegar al siguiente eslabón será necesario pasar por el previo; principio que define al método proyectual, provechoso por mucho tiempo para el ejercicio del diseño, pero circunscrito dadas las características del contexto. El método proyectual ha perdido aptitud frente a problemas y dificultades propias del siglo XXI, donde las condiciones y dinámica se caracteriza por la yuxtaposición de una variedad de fenómenos de naturaleza heterogénea (Costa, 2009, p.10). Esto requiere ir más allá de un intersticio, apuntando hacia perspectivas más amplias sostenidas en la multidisciplinariedad (Tiburcio, 2015, p.229), donde intervienen factores heterogéneos, 
multidimensionales y otros de diversa naturaleza, los cuales a través de una equilibrada complejidad sistémica dan forma al fenómeno del diseño.

Frente a esta coyuntura, estimulada por la asimilación dada en las ciencias sociales sobre la Teoría General de los Sistemas, difundida originalmente en 1947 por Bertalanffy (1986), ampliada por los aportes de Luhmann (1991) y consolidada luego en la Teoría de los Sistemas Complejos Adaptativos de Holland (2004), se promovería un enfoque epistemológico distinto, adoptado por áreas del diseño que traerían nuevas estructuras de pensamiento, a partir de métodos de diseño de perfil sistémico. Dicho planteamiento, opuesto a la visión cartesiana, incentiva la capacidad creativa (Briede, Leal y Pérez, 2018, p.11). De esta manera, es posible dilucidar que una persona formada en diseño requiere entender los problemas más allá de la causalidad, dentro de lo cual se presenta la complejidad como una opción, donde los métodos de diseño emergen como instrumento relevante en dicha transición. En el caso mexicano, destaca el desarrollo de métodos de diseño tales como el Modelo Diana, propuesto por Oscar Olea y Carlos González, así como el Modelo General de Procesos de Diseño, propuesto por la Universidad Autónoma Metropolitana (México).

En lo referente a la práctica del diseño y la aplicación de métodos en el ejercicio profesional, Castells (2005, p.141) explica cómo el contexto laboral del siglo XX se concebía a través de espacios físicos para la producción. Sin embargo, a partir de la masividad de la tecnología digital, se transitaría poco a poco hacia un nuevo paradigma, donde los intercambios e interacciones serían inconexos físicamente. Esto ha transfigurado la práctica profesional del diseño hacia una conectividad cada vez más digital y menos personal.

En este escenario laboral, las personas diseñadoras enfrentan la división nacional e internacional de trabajo, lo cual sobresale como variable sin precedente (Castells, 2005, p.148). En esto, una persona diseñadora debe ser un sujeto profesional que funja principalmente como agente articulador de la creatividad y la innovación en las empresas (Dorochesi, 2010, p.95), lo que lo empuja a emigrar de lo que tradicionalmente ha definido su actividad al mundo de las ideas como centro de su quehacer profesional. Quienes han adoptado el nuevo rol han sido más capaces de influir en la solución de problemas y de las necesidades sociales (Tiburcio-García, 2013, p.77), lo que se ha traducido en más y mejores clientes.

No obstante, para incentivar la transición a las ideas, resulta fundamental promocionar la capacidad analítica y de innovación en una persona diseñadora desde nuevos enfoques, para lo cual destacan los métodos como engranaje relevante. Dicha responsabilidad recae en 
la formación universitaria, algo que requerirá de compromiso y acción, pues como advierte Pimentel (2010, p.223), las nuevas personas diseñadoras se siguen formando con planes obsoletos que obedecen a otras realidades, lo que exhorta a las universidades a buscar estrategias que permitan reenfocar la formación de profesionales aptos para la dinámica laboral y sus exigencias.

Con respecto a la aplicación y uso de los métodos de diseño en el ejercicio profesional en México, el estudio de Morales y González (2020, p.41) ofrece una aproximación. En este se estima que más del $50 \%$ de las personas diseñadoras utilizan el método lineal, mientras una menor proporción se inclina por el método sistémico. Por otro lado, casi un $20 \%$ apuestan por la creatividad en lugar de un método, enfoque sustentado en la intuición natural y que inspiraría procesos para diseñar, los cuales se pueden definir como innatos y carentes de una estructura formal, propios de etapas previas a las escuelas pioneras de principios del siglo pasado. Lo anterior devela que un segmento importante de las personas diseñadoras, en pleno siglo XXI, emplea la intuición como método, lo que se asume es un riesgo de cara a la complejidad ya descrita, y que demanda el entorno.

\subsection{Enseñanza del diseño y su desarrollo en México}

Desde los inicios, con la Escuela de la Bauhaus, la enseñanza del diseño se ha basado en la estrategia de taller, modelo pedagógico que se sustenta primordialmente en el ejercicio práctico y poco en la investigación. La enseñanza en el taller se fundamenta en el estudioaprendizaje basado en los haceres (Tovey, 2015, p.1), proceso guiado por el profesorado, quien simula proyectos reales que el alumnado busca resolver a través de sus productos creados, sus diseños para desarrollar competencias y conocimientos técnicos (Rodríguez, 2016, p. 256).

De esta manera, la enseñanza del diseño se centra en algo parecido al desarrollo de un oficio, donde importa la práctica que el estudiantado desarrolle y las competencias que requerirá para su actividad profesional. La adquisición de experiencia es el principal objetivo; se busca que los productos sean resultado de la crítica y la reflexión, lo que demanda una base metodológica, además de una capacidad de interconexión de realidades heterogéneas y convergentes.

En busca de soluciones posibles aplicables al campo del diseño, se considera viable recuperar el planteamiento de Díaz Barriga (2006, p.9), quien propone, de manera práctica, qué tipo de didáctica convendría seguir en la enseñanza, al destacar que en los cursos que 
incluyen proyectos, más allá de enseñar a crear objetos o conceptos visuales, es necesario centrarse en enseñar cuáles objetos son los que conviene diseñar; es decir, la capacidad de innovar. No obstante, los métodos destacan como aspecto de suma relevancia en el campo del diseño y la formación universitaria, por lo que es importante destinar un espacio a reflexionar sobre estos (Rivera Díaz, 2018, p.44), pues resultan determinantes como promotores del diseño.

Desde una perspectiva más general, algunas investigaciones evidencian de forma teórica y empírica el rol de la edad del docente en la enseñanza. Por ejemplo, Zempoalteca, González, Barragán y Guzmán (2018) señala que atributos como la edad, se asocian con el uso de la tecnología en la enseñanza. Por su parte, Hernández, Partido y Hernández (2009) precisan que la edad del profesorado influye en su actuar, y después de algunos años de estar en la docencia, se condicionan sus actitudes y el desempeño profesional. Advierten que se requiere impartir cursos de formación teórica-práctica al profesorado más joven, de modo que se evite el aprendizaje por ensayo y error. Asimismo, de las diferencias significativas halladas por Feixas (2010), se sustenta que a mayor edad y años de experiencia en la docencia universitaria, hay mayor protagonismo en la enseñanza, dando forma a un profesorado que principalmente ejerce la transmisión de contenido y en su conocimiento sobre la materia. Esto a diferencia de una persona joven que se desempeña en la docencia, quienes manifiestan lagunas de conocimiento en materias que imparten y requiere sugerencias para su desarrollo.

Otros estudios como el de Madrid (2005) sostienen que la edad a veces influye en la metodología de enseñanza que es empleada por el profesorado. Mientras algunas personas valoran más la experiencia docente para definir sus métodos y estrategias, el personal docente más joven tiene mayor entusiasmo e ilusión para motivar al estudiante. Esta misma idea se relaciona con el estudio de García-Rangel y García, Reyes (2014), quienes argumentan que el alumnado siente mayor empatía con el personal docente más joven. Por su parte, el trabajo el de Stes, Gijbels y Van Petegem (2008) identifican, a través de métodos estadísticos, una relación de la edad con diferentes aspectos de la enseñanza (enfoques docentes y la disciplina).

Además, un acercamiento inicial a esta situación enfocada en el diseño, es el estudio de Garone Gravier (2011), ya señalado, el cual explora la lucha generacional entre diseñadores "analógicos" y "digitales", donde la edad se presenta como factor preponderante. Por su parte, la investigación de Morales y González (2020), analiza el uso de los métodos de diseño de 
acuerdo con el grado académico y experiencia, donde teóricamente la edad se vuelve factor de la enseñanza y la práctica profesional.

Al indagar sobre que se ha hecho en este sentido, queda en manifiesto que dicha intensión ha sido insuficiente, pues sus modelos de enseñanza-aprendizaje y los métodos para diseñar parecen manifestar un atemporal desarrollo y aplicación con respecto al rápido ritmo que caracteriza al campo del diseño. Asimismo, se ha creado la necesidad de adaptarse a la realidad actual, pues algunas de las herramientas que funcionaron en el pasado resultan obsoletas ahora, lo que lleva al diseño como disciplina y a las personas diseñadoras en su práctica, a la necesidad de adaptación y constante actualización. Este fenómeno cultural y económico, dados sus alcances y repercusiones, obliga a reflexionar en él e investigarlo (Ramírez, Villar y Maldonado, 2017, p. 54).

En ese sentido, convendría apostar por una enseñanza que rebase los conocimientos básicos y fundamentales (expresión gráfica, historia del arte, dominio de software, composición gráfica, etc.) por otros que capaciten para dilucidar y operar los problemas desde una perspectiva compleja, como un sistema de pensamiento. Se trata de una mejor formación que incorpore, de forma paralela, un enfoque promotor de las capacidades cognitivas, para lo cual aspectos teóricos, la lectura y la escritura actuarían de forma vinculada con los talleres de diseño tradicionales, como generadores del pensamiento crítico (Rivera, 2018, p.161).

Los planes de estudio del área de diseño de las principales universidades en México se fundamentan en el sistema de taller, que ha resultado ser un importante promotor de sus habilidades. No obstante, al mismo tiempo se evidencia la necesidad de enfocarse en la teorización, la cual en ciertos casos es conformada por una estructura de materias que suman un número inferior a las del taller, por conjuntos reducidos de asignaturas, así como por solo un mínimo de asignaturas aisladas. También se identifica que algunos programas de estudio presentan un ordenamiento poco claro de los conocimientos que se imparten, a veces incongruentes con la realidad profesional. En conjunto, son aspectos que podrían contribuir, junto con la dispersión por multitareas, en la promoción de aquella inercia, la cual mina el conocimiento y fomenta la búsqueda de alternativas de trabajo prácticas o que demanden menor tiempo de atención.

Para Pérez Gómez (2013, p. 66), la dispersión por multitareas es el fenómeno que ocurre en quienes se encuentran saturados de información y de exigencias al momento de permanecer en conexión con Internet. Es resultado de asumir el desempeño de múltiples tareas a realizar de forma simultánea —-mayormente digitales_ para dedicar atención parcial 
a cada actividad; dispersión que desorienta el conocimiento e incrementa la ansiedad. Esta coyuntura empuja a buscar alternativas prácticas que demanden menor tiempo y atención, ingresando así, de manera consciente o no, a una inercia de optar por esfuerzos menores.

Ante los problemas descritos se presenta como necesario, una mayor estimulación de los métodos de diseño, pues juega un papel relevante como promotor del pensamiento, del análisis y las ideas. De acuerdo con esto, Rivera Díaz (2018, p.61) destaca que tres conceptos deben interactuar en la búsqueda de una didáctica proyectual, cuyo objetivo sea el desarrollo de competencias para un pensamiento crítico del diseño: a) una situación problemática, contenida en el proyecto (análisis y crítica); b) una estructura cognitiva, capaz de activarse para la resolución de problemas, y c) ambas. En la misma dirección, Ramírez, Villar y Maldonado (2017, p.4) concuerdan en que dicha formación estimula la transición del rol tradicional de personas profesionales del diseño (limitado a la reproducción) al de la innovación. En ese sentido, constituir para las personas diseñadoras, una formación que incluya destrezas cognitivas, la promoción de una visión integral de los problemas y situaciones, así como una visión prospectiva, dependerá primordialmente del profesorado, al ser los promotores del cambio en las universidades. Por ello, su formación y estilo de pensamiento, así como la edad, parecen jugar un papel significativo.

En relación con la situación expuesta, el propósito del texto es identificar diferencias en el uso de los métodos de diseño que se utilizan en la enseñanza y la práctica profesional según la edad del profesorado universitario en México, al ser un sujeto que estimula y guía el pensamiento de las personas diseñadoras a lo largo del proceso creativo. Mediante esto se podrá incitar y promover un mejoramiento de la formación del diseño.

\section{Metodología}

\subsection{Enfoque}

El enfoque de esta investigación fue cuantitativo, para indagar tendencias, actitudes $u$ opiniones entre una población mediante el estudio de una muestra (Creswell, 2009, p. 145). Por lo tanto, se diseñó una investigación de cobertura nacional en cuanto a su alcance, se incluyó a instituciones como: Universidad Autónoma Metropolitana, Universidad Autónoma de Baja California, Universidad Iberoamericana, Universidad Autónoma de Nuevo León, Universidad de Sonora, Universidad Autónoma de ciudad Juárez, Universidad de Guadalajara y otras, bajo el método exploratorio-descriptivo como estrategia metodológica. 


\subsection{Unidades de análisis}

La investigación iniciada desde el año 2018, denominada Prospectiva del campo profesional del diseño en las IES de México: formación, pensamiento y ejercicio profesional, contempló universidades de 12 estados (Baja California, Chihuahua, Ciudad de México, Jalisco, Nuevo León, Sonora, Sinaloa, y otras), e incluye a las principales universidades que ofertan en su formación al diseño. No obstante, a pesar de las limitaciones de la muestra de respuestas obtenidas, se recuperó una representación importante que permite un acercamiento a la realidad referente al uso de los métodos de diseño en la enseñanza y la práctica profesional en México, de acuerdo con la edad del profesorado. El estudio y los resultados que se analizan en este artículo, con base en la percepción del profesorado que labora en las principales universidades de México señaladas, consideró un muestreo no probabilístico, por conveniencia, el cual fue realizado para obtener respuestas de sujetos que estuvieron dispuestos a participar a partir de su interés de colaborar (Marradi, Achenti, y Piovani, 2010). Como criterio de inclusión, se determinó el cumplir con desempeño de actividades de docencia en alguna IES de México dentro del campo del diseño, así como su rango de edad, el cual se estructuró en tres grupos: menores de 40 años, que incluyó 46 sujetos; de 41 a 60 años, con una participación de 61 sujetos; y mayores de 61 años, con 19 sujetos.

\subsection{Técnicas de recolección de información}

El instrumento utilizado para el proceso de recolección de datos fue un cuestionario y su aplicación se hizo en formato digital, el cual se logró distribuir a través de correos electrónicos. Esto buscó indagar la percepción del profesorado a través de 42 reactivos vinculados a diferentes dimensiones: estrategias de enseñanza y contenidos curriculares, la perspectiva interdisciplinar en la formación, condiciones académicas, uso de métodos de diseño, entre otros aspectos. El trabajo de campo durante tres meses (enero-marzo, 2019), permitió la recopilación de una muestra de 126 sujetos, académicas y académicos adscritos a diferentes universidades públicas (Universidad Autónoma Metropolitana, Universidad Autónoma de Nuevo León, Universidad de Guadalajara, Universidad Autónoma de Ciudad Juárez, entre otras.) y privadas (Universidad Iberoamericana, Universidad Lasalle, entre otras). Del total de personas encuestadas, el $77 \%$ fueron del primer tipo de universidades y un $23 \%$ del segundo (privadas). La experiencia académica del profesorado figura en un rango de entre 1 a 46 años. En cuanto a las edades, el 48\% oscila entre los 41 y 60 años, un $15 \%$ son 
mayores de 61 años, mientras que un 37\% corresponde a personas menores de 40 años (Tabla 1). En cuanto a sexo, el $51 \%$ son hombres y el $49 \%$ mujeres; $42 \%$ son de categoría de contratación de tiempo completo (desempeñan 40 horas a la semana) y $58 \%$ con contrato como personal docente de asignatura; 70\% tiene posgrado (maestría o doctorado).

\subsection{Procesamiento de análisis}

La información recopilada tuvo un tratamiento de tipo analítico con base en técnicas de estadística descriptiva a través el programa SPSS 24 (Statistical Package for the Social Sciences) y presentada mediante gráficos que fueron elaborados a través de la herramienta Microsoft office Excel. Estos datos fueron procesados desde un enfoque organizativo de acuerdo con dimensiones definidas, según tres rangos de edades (menores de 40 años, 41 a 60 años y mayores de 61 años).

En función del planteamiento expuesto, este texto aborda específicamente tres variables ubicadas en la dimensión de uso de métodos de diseño, resultado de diferentes niveles de delimitación del dato. Las variables relacionadas con la utilización de métodos de diseño son: 1) para enseñar, 2) en el ejercicio profesional y 3) al diseñar. De forma adicional, se utilizó la edad como variable independiente para comprobar tendencias en el uso de métodos de diseño, con el propósito de establecer explicaciones basadas en datos empíricos (Marradi, et al., 2010, p. 247).

Para detallar acerca de los reactivos correspondientes a los métodos de diseño, las principales opciones de respuestas fueron: lineal-secuencial y sistémico (complejidad), aunque también se consideró lo que se denominó creatividad pura, a pesar de que la creatividad como tal no se erige desde un andamiaje epistémico para valorarse como método de diseño en sí, más bien a partir de la subjetividad creativa. Sin embargo, su tradición en la enseñanza y aplicación en la práctica, aunque cuestionada, promueve dicha inclusión. Asimismo, se retomó como opción: otros modelos, la cual se conformó por métodos distintos a los antes mencionados.

Además, se aplicó una prueba de hipótesis estadísticas (Chi-cuadrada) para comprobar si hay diferencias estadísticamente significativas en el uso de métodos de diseño con respecto a la edad. 


\section{Resultados y discusión}

Los resultados fueron ordenados a partir de tres criterios: 1) método de diseño para enseñar diferenciados por la edad de sujetos; 2) métodos de diseño más utilizados en el ejercicio profesional diferenciados también por la edad; 3) métodos de diseño utilizados para diseñar diferenciados por la edad del profesorado.

\subsection{Método de diseño para enseñar diferenciados por la edad del profesor}

Los resultados presentados en la Figura 1 permiten observar que a mayor edad del profesorado, se opta por el método sistémico, mientras a menor edad se inclina por el método lineal. En cuanto a aquellas personas menores de 40 años, se identificó que tiene mayor dominio el método lineal-secuencial (69\%), paralelamente utilizan en menor proporción el método sistémico (20\%). En forma diferenciada, el profesorado de 61 años en adelante, son los que se inclinan en mayor medida por el método sistémico (74\%), al mismo tiempo, fue el grupo que desdeñó en superior medida el método lineal (16\%). En un segmento intermedio se ubicó al profesorado de entre 41 y 60 años, grupo que arrojó resultados más próximos a los del grupo de mayor edad, que optan por el método sistémico como el adecuado para la formación (57\%), y en menor medida al método lineal-secuencial (23\%). En lo referente a otros modelos, los tres grupos asignaron valores equivalentes, entre (11\%) y (13\%). La creatividad pura solo fue considerada por el grupo que comprende edades de 41 a 60 años (7\%). 
Figura 1

México: Métodos de diseño que se utilizan para enseñar según la edad de la persona docente, periodo 2018-2019.

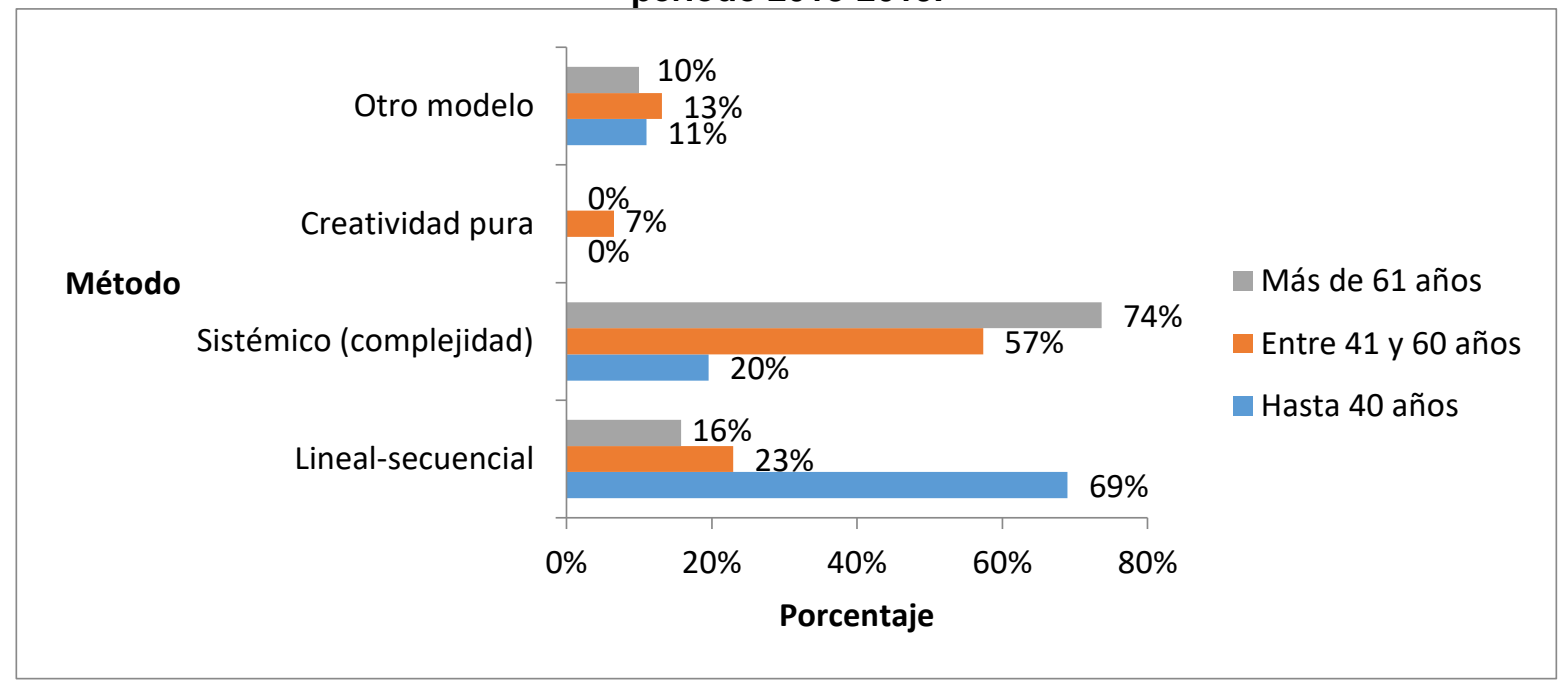

Fuente: Elaboración propia, según los datos recopilados.

Los datos arrojados muestran que existen diferencias entre el profesorado, divididas por el rango de los 40 años. Aquellos ubicados con mayor edad, (41 a 60 años y 61 o más años) exhiben una visión con respecto al proceso de diseño a partir de sus métodos, con disposición a la complejidad, y se asume su fenomenología a partir de procesos diversos y la interacción de múltiples fenómenos, andamiaje propio de la propuesta sistémica. De forma diferenciada, el profesorado con una edad de 40 años o menos, dilucida sobre el diseño y sus procesos de creación a partir de la causalidad, al recoger la visión cartesiana como soporte teórico-práctico.

De las tendencias manifiestas, según los datos analizados, se infiere que la edad es una variable preponderante en la elección del método de diseño que una persona designa para la enseñanza, lo cual permite suponer que el personal docente formado en la etapa digital, quienes fueron rodeados de mayor tecnología, acceso multimodal a Internet, así como fácil acceso a la información, apuestan por la propuesta tradicional causal. En cambio, el profesorado mayor de 40 años, en especial aquellos próximos a los 60 años, formados en un entorno mayoritariamente predigital, fue el grupo que manifestó estar al tanto de las tendencias y propuestas teóricas de vanguardia, al apostar claramente por la visión sistémica, a pesar de que el andamiaje haya sido abordado recientemente por la investigación en diseño.

Adicionalmente, se encontró una asociación significativa de los rangos de edad del profesorado con los métodos de diseño que se utilizan para enseñar (Chi-cuadrada = 33.99, $\mathrm{p}=0.001$ ). De esta amera y de acuerdo con los resultados, la edad se presenta como factor 
significativo en cuanto al uso de los métodos de diseño, donde los 40 años de edad actúa como línea divisoria de ambas perspectivas entre los diseñadores.

\subsection{Métodos de diseño más utilizados en el ejercicio profesional diferenciados por edad del profesorado.}

La información arrojada en la Figura 2, muestra cómo el profesorado menor de 40 años considera que el método lineal es, en la práctica profesional, el que más utilizan las personas diseñadoras (67\%), mientras encuentran el método sistémico en menor proporción (20\%). En rangos menores se encontró la creatividad pura (11\%) y a otros modelos (2\%). Desde otra perspectiva, las opiniones de aquellas personas en edades de entre 41 y 60 años consideraron de manera próxima el uso de los métodos lineal (31\%), sistémico (36\%) y creatividad (26\%) como los más utilizados por las personas diseñadoras, otros modelos representaron una menor parte (7\%). Quienes se ubican en el rango de 61 años en adelante, consideran al método lineal como el de mayor aplicación (42\%), seguido del sistémico (32\%), creatividad (21\%) y otro modelo (5\%).

Figura 2

México: Métodos utilizados en el ejercicio profesional del diseño según la edad de la persona docente, periodo 2018-2019.

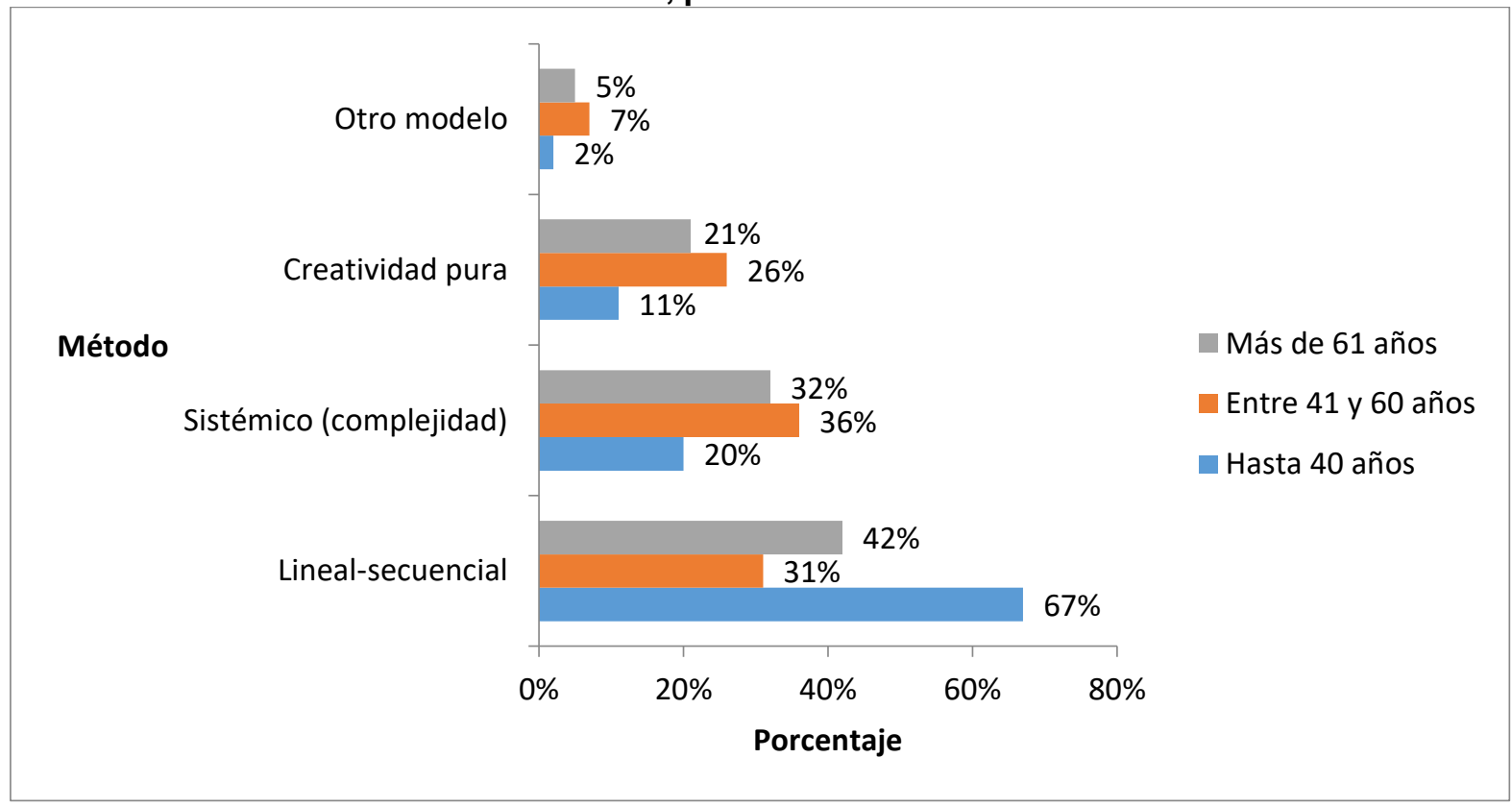

Fuente: Elaboración propia, según los datos recopilados. 
Los resultados manifiestan promedios similares entre los grupos con edades superiores a los 40 años; mientras el grupo con el rango de edad debajo de este expuso otros datos. El personal docente con edad superior a los 40 años, reflejan valores claramente distintos entre ambos reactivos (Figura 1 y 2), al elegir el método sistémico para la enseñanza, mientras entienden que lo que se ejerce en el campo profesional apunta hacia el empleo de los métodos lineal, sistémico y creatividad pura en rangos más o menos equivalentes. Este resultado exhibe un análisis individual desarrollado para cada contexto.

Por su parte, el grupo comprendido por edades de hasta los 40 años prácticamente replicó los promedios, resultado de las opiniones que fueron vertidas en el actual reactivo (Figura 2) y el anterior (Figura 1). La replicación de las cifras dadas en ambas gráficas con respecto a los métodos lineal y sistémico se asume que podría ser un reflejo de la inercia por esfuerzos menores, influido por la citada dispersión por multitareas que parece ser característica de esa generación, lo que a su vez pudiera promover una deficiencia en la crítica de hechos, sin que esto sea producto de escasez de capacidad. Más bien se podría atribuir a aquella conducta hacia la practicidad y las respuestas rápidas, consecuencia de la saturación y dispersión de tareas que, se asume, influyen en su dinámica conductual.

Desde otra perspectiva, en cuanto a la creatividad, a pesar de no ser un método de diseño, según opiniones de personas con 41 años o más, un 24\% de las personas diseñadoras desarrollan su trabajo sin el sustento de un método, solo a partir de la intuición. Esto resulta preocupante, pues se manifiesta que una cantidad considerable (aproximadamente 25\%) del diseño se erige sin el soporte de un método, lo que cuestiona severamente su calidad y pone en entredicho el prestigio de la persona docente y de las universidades.

Apoyando los resultados descritos, se identificó una diferencia significativa en los rangos de edad del personal docente con los métodos de diseño que se utilizan en el ejercicio profesional (Chi-cuadrada $=40.70, p=0.001$ ). Como resultado se identifica la edad como elemento significativo en el empleo de los métodos de diseño en la práctica profesional, emergiendo dos rutas conductoras, la que apoya a los métodos progresistas y la que se apega a los tradicionales, donde se presenta, una vez más, los 40 años como variable que marca tendencias diferentes. 


\subsection{Métodos de diseño utilizados para diseñar diferenciados por la edad del profesorado.}

Al cuestionar sobre el método de diseño que el profesorado aplica al momento que diseñan (Figura 3), de igual manera, los resultados fueron divididos en dos conjuntos principales, a partir de la línea de los 40 años. Los ubicados debajo de dicha línea optan por el método lineal, mientras el grupo situado sobre esta prefiere el método sistémico.

El personal docente que conforma el grupo menor de 40 años apuestan por el método lineal en mayor proporción (58\%), luego por el sistémico (24\%) y dan a la creatividad y a otros modelos valores similares (9\%). Aquellos de 41 a 60 años se inclinan por el método sistémico (64\%), otros modelos (16\%), creatividad pura (12\%) y método lineal (8\%). El grupo de más de 61 años también optó por el método sistémico (53\%), luego consideraron la creatividad pura (26\%) y, en un mismo rango, la linealidad y otros modelos (11\%).

Figura 3

México: Métodos que el profesorado utiliza al diseñar según la edad de la persona docente, periodo 2018-2019.

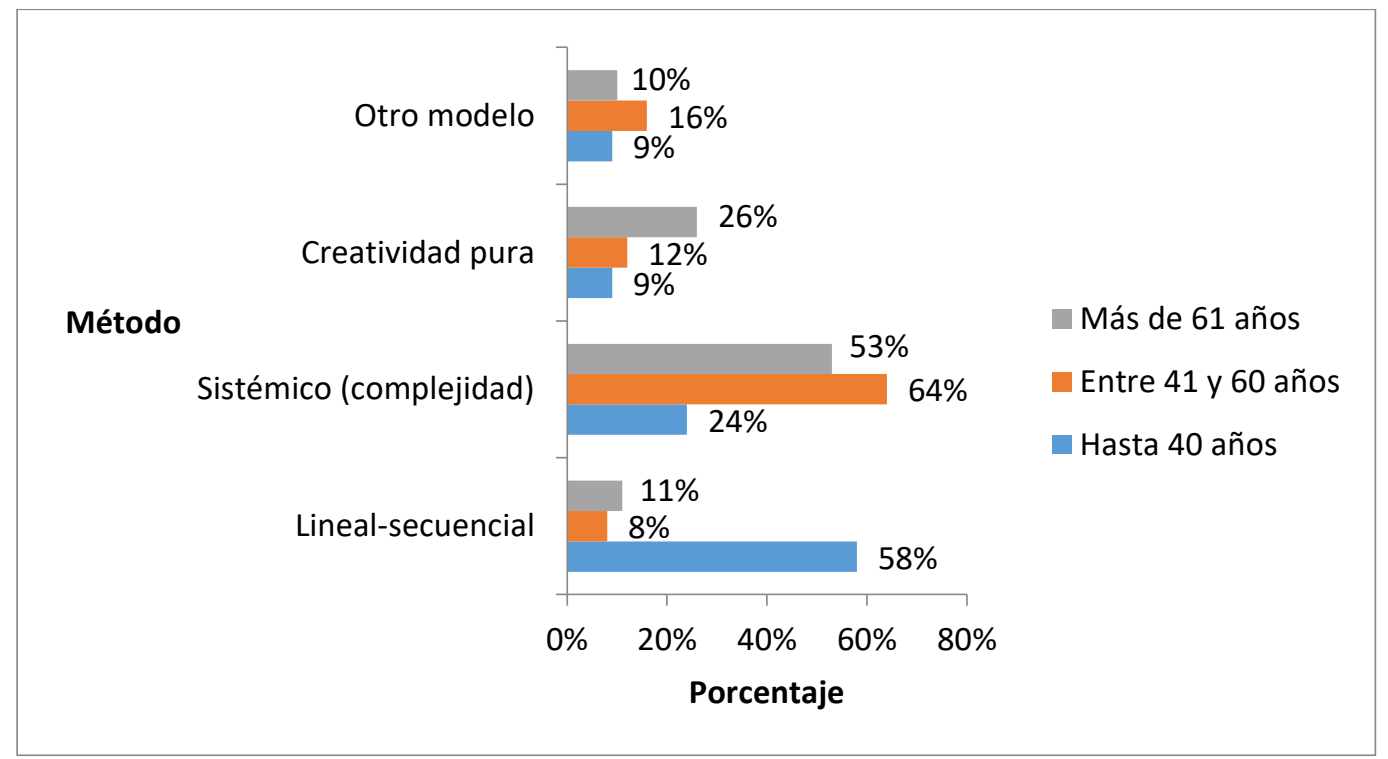

Fuente: Elaboración propia, según los datos recopilados.

Los datos reflejan que el grupo de sujetos de entre 41 y 60 años es el que opta en mayor medida por el método sistémico a la hora de diseñar; aunque aquellos con 61 años o más lo eligieron en número ligeramente inferior. Con ello se reafirma la homogeneidad de enfoque entre ambos conjuntos. 
De manera opuesta, el personal docente menor de 40 años se inclina por el método lineal de forma más clara (58\%), seguido a la distancia por el método sistémico (24\%). Esto deja en evidencia que el profesorado más joven, formados en la etapa tecnológica-digital, se decantan por modelos concebidos como obsoletos, propios del siglo anterior; mientras aquellas personas de mayor edad, educadas en una etapa caracterizada por un entorno tecnológico limitado, ausentes de herramientas como Internet, han dejado de lado los métodos de diseño convencionales con los cuales fueron formados en un principio, al optar por aquellos categorizados como vanguardistas.

Finalmente, el análisis de datos permitió ubicar una diferencia significativa entre los rangos de edad del profesorado con los métodos de diseño que utilizan al diseñar (Chicuadrada $=14.23, p=0.027$ ), por lo que la edad, en la misma tesitura, contribuye significativamente en la elección del método, donde se mantiene los 40 años de edad como variable conductual relevante entre ambos grupos de diseñadores.

\section{Conclusión}

Se analizó el uso de métodos para diseñar en relación con la enseñanza y práctica profesional, desde la perspectiva del profesorado que se desempeña en diversas áreas de diseño en México. Este análisis recupera tendencias según las edades, característica del sujeto que se asume como un reflejo de una formación recibida, la cual es diferenciada por dos contextos: predigital y digital. Así, el estudio tomó como referencia a las principales universidades que cuentan en su perfil de formación al diseño, y aunque permite una aproximación importante que ofrece pautas sobre la situación, los resultados están alejados de una posible generalización en la realidad de todas las IES del país.

En torno a esto, se identificó una divergencia de opinión entre el profesorado con edad de hasta 40 años y quienes superan dicho rango. Los primeros, formados en un entorno tecnológico, de la información y uso generalizado de Internet, conciben el campo del diseño a partir de una estructura causal y abordan su desarrollo desde métodos lineales-secuenciales, propios del siglo XX. Desde otra perspectiva, las personas docentes con rango de edad que van de los 41 años en adelante, de los cuales una parte supera los 60 años y están formados en un contexto caracterizado por limitada tecnología, poco acceso a la información y, en muchos casos, con ausencia de Internet, mostraron una perspectiva más amplia e incluyente sobre el diseño, al asumirlo como un fenómeno complejo, perspectiva apoyada en la interdisciplina y el método sistémico como enfoque viable para su desarrollo. 
De tal manera, se concluye que, a menor edad del profesorado, se opta por modelos y métodos de diseño menos actualizados y obsoletos, mientras a mayor edad se apuesta por métodos prospectivos, la actualización y la vanguardia, tanto en la enseñanza como en la práctica. Tal divergencia parece obedecer, por un lado, a un nivel de estudios más elevado de las personas de mayor edad frente a los más jóvenes; y, por otro lado, a la degradación de la calidad académica en diseño (Morales y González, 2020, p.41), en concreto en aquellos formados en la etapa digital, la cual parece haber sido rebasada por la naturaleza de un entorno profesional siempre cambiante y caracterizado por la incertidumbre. Aunado a esto, está la dispersión por multitareas, propia de una etapa más digital, donde la saturación de información, tareas y exigencias incentivan la ansiedad y minan el conocimiento, lo cual se traduce en decisiones y conductas en pro del menor esfuerzo y en el menor tiempo posible.

Por lo tanto, según los resultados obtenidos y sin perder de vista las limitaciones de la muestra obtenida, la formación de profesionistas del diseño en México tiende a erigirse a partir de dos perfiles docentes: el de menor edad, con amplio conocimiento y dominio tecnológicodigital, pero con menor capacidad de dilucidar al diseño desde una perspectiva integral, privilegiando así la técnica sobre el análisis y el razonamiento; y aquellos de mayor edad y mayor nivel de estudios, portadores de un criterio más amplio y visión prospectiva, quienes valoran e incentivan el análisis y la crítica en la formación de personas diseñadoras.

De acuerdo con las necesidades que demanda el contexto actual, donde destacan conocimientos de un orden cada vez más elevado y enfoques metodológicos más amplios e integrales, la generación de profesoras y profesores jóvenes requerirá adoptar estrategias y perspectivas nuevas, con nuevas teorías y una cultura de constante aprendizaje multidisciplinar que trascienda la técnica, y así superar obstáculos como la cultura del menor esfuerzo, la poca prioridad otorgada a los métodos de diseño y su trascendencia, la valoración de Internet y sus contenidos como la panacea, así como la reverencia de la tecnología como soporte capital del diseño. De lo contrario, se corre el riesgo de caer en la degradación de la formación del diseño al ejercicio técnico. En ese sentido, los cambios se muestran necesarios, debido a que el diseño es un campo que va más allá de solo las cuestiones tecnológicas y que se caracteriza por transformaciones constantes y una rápida obsolescencia.

Lo anterior deja al descubierto que una formación que aparentemente es enriquecida con todos los avances tecnológicos e información no necesariamente se traduce en una formación profesional de vanguardia, ni en planes de estudio de avanzada que promuevan el razonamiento y análisis. Asimismo, esto no necesariamente se traduce en innovación. De 
esta manera, emerge un ejercicio autocrítico y necesario, el cual permita acceder a mejores estrategias de formación y a delinear mejores políticas educativas a favor de la enseñanza del diseño.

\section{Referencias}

Bertalanffy, Ludwig Von. (1951). General system theory, a new approach to unity of science. Human biology, 23(4), 337-345.

Bertalanffy, Ludwig Von. (1986). Teoría general de los sistemas. Ciudad de México, México: Fondo de cultura económica.

Bertola, Paola. (2004). El diseño como herramienta de investigación: nuevas oportunidades para las competencias del Diseño. En S. Mac Donald (Ed.), Temas de diseño en la Europa de Hoy (pp. 36-38). Barcelona, España: BEDA. Recuperado de https://www.academia.edu/16672698/TEMAS DE DISE\%C3\%910 EN LA EUROPA DE HOY

Briede-Westermeyer, Juan., Leal-Figueroa, Isabel., y Pérez-Villalobos, Cristhian. (2018). Análisis de Referentes como Estrategia de Aprendizaje del Diseño Conceptual de Productos. Formación universitaria, 11(1), 3-12. doi: http://dx.doi.org/10.4067/S0718$\underline{50062018000100003}$

Castells, Manuel. (2005). La era de la información: economía, sociedad y cultura. La sociedad red. Madrid, España: Alianza Editorial.

Castells, Manuel. (2009). Comunicación y poder. Madrid, España: Alianza Editorial.

Costa, Joan. (2009). El DirCom hoy: Dirección y Gestión de la Comunicación en la nueva economía. Barcelona, España: Costa Punto Com.

Cramer-Petersen, Claus., Christensen, Bo., y Ahmed-Kristensen, Saeeman. (2019). Empirically analysing design reasoning patterns: Abductive-deductive reasoning patterns dominate design idea generation. Design Studies, 60, 39-70. doi: https://doi.org/10.1016/i.destud.2018.10.001

Creswell, John. (2009). Research design: Qualitative, quantitative, and mixed methods approaches. Thousand Oaks, Estados Unidos: Sage.

Díaz Barriga, Frida. (2006). Enseñanza situada: vínculo entre la escuela y la vida. Ciudad de México, México: McGraw Hill.

Dorochesi Fernandois, Mario. (2010). Complejidad, creatividad y cambio. Reflexiones en torno a los nuevos escenarios para la enseñanza del Diseño. Actas de Diseño, 5(9), 95-101. Recuperado https://fido.palermo.edu/servicios dyc/publicacionesdc/archivos/148 libro.pdf 
Feixas, Mónica. (2010). Enfoques y concepciones docentes en la universidad. RELIEVE: Revista Electrónica de Investigación y Evaluación Educativa, 16(2), 1-27. Recuperado de https://ojs.uv.es/index.php/RELIEVE/article/viewFile/4134/3754

García-Rangel, Edna Guadalupe., García Rangel, Ana Karenina., y Reyes Angulo, José Antonio. (2014). Relación maestro alumno y sus implicaciones en el aprendizaje. Ra Ximhai, 10(5), 279-290. Recuperada de https://www.redalyc.org/pdf/461/46132134019.pdf

Garone Gravier, Marina. (2011). Textos y contextos de una década de diseño gráfico en México (1990-2000). Ensayos: Historia y Teoría del Arte, (21), 77-120.

Hernández, Griselda., Partido, Hilda., y Hernández, Edith. (2009). La efectividad de la enseñanza y su relación con la edad de los enseñantes. aproximaciones a las percepciones de los estudiantes universitarios. Presentado en el $X$ Congreso Nacional de Investigación Educativa. Veracruz, México. Recuperado de http://www.comie.org.mx/congreso/memoriaelectronica/v10/pdf/area tematica 14/pone ncias/1734-F.pdf

Holland, John. (2004). El orden oculto, de cómo la adaptación crea la complejidad. Ciudad de México, México: Fondo de cultura económica.

Luhmann, Niklas. (1991). Sistemas sociales: lineamientos para una teoría general. Ciudad de México, México: Alianza Editorial; Universidad Iberoamericana.

Lupton, Ellen., y Miller, Abbott. (2002). El ABC de la Bauhaus y la teoría del diseño. Barcelona, España: Gustavo Gili.

Madrid, Daniel. (2005). La edad del profesorado en los procesos de enseñanza y aprendizaje de la lengua extranjera. En J. Martínez Dueñas, C. Pérez Basanta, N. McLaren y L. Quereda, Towards an understanding of the English Language: Past, Present and Future. Studies in Honour of Fernando Serrano (pp.519-530). Granada, España: Editorial Universidad de Granada.

Marradi, Alberto., Achenti, Nélida., y Piovani, Juan Ignacio. (2010). Metodología de las Ciencias Sociales. Buenos Aires, Argentina: Cengage Learning.

Meggs, Philip. (2010). Historia del diseño gráfico. Ciudad de México, México: Trillas.

Morales-Holguín, Arodi., y González-Bello, Edgar. (2020). Enseñanza y uso de métodos de diseño en México. Percepciones del profesorado. Formación universitaria, 13(1), 3542. doi: https://dx.doi.org/10.4067/S0718-50062020000100035

Morin, Edgar. (2004). Introducción al pensamiento complejo. Barcelona, España: Editorial Gedisa.

Moreno Toledano, Leonardo., y Rogel Villalba, Erika. (2012) Retrospectiva del método en el diseño. En S. V. Ariza Ampudia (Ed.), La investigación en diseño, una visión desde los 
posgrados en México (pp. 79-109). Ciudad Juárez, México: Universidad Autónoma de Ciudad Juárez.

Nacif, Nora. (2015). Métodos de diseño. Apuntes de Cátedra: métodos y estrategias de diseño. Recuperado de http://www.faud.unsj.edu.ar/descargas/blogs/apuntes-de-ctedramtodos-y-estrategias-dediseo Metodos\%20y\%20Estrategias\%20de\%20Dise\%C3\%B10.pdf

Pérez Gómez, Ángel. (2013). La era digital. Nuevos desafíos educativos. Revista Electrónica Sinéctica, (40), 47-62. https://www.redalyc.org/pdf/998/99827467010.pdf

Recuperado de

Pimentel Jiménez, Julia Virginia. (2010). Formando diseñadores de interiores del Siglo XXI con planes de estudio del Siglo XX. Actas de Diseño, 5(9),9, 223-226. Recuperado de https://fido.palermo.edu/servicios dyc/publicacionesdc/archivos/148 libro.pdf

Ramírez-Paredes, Karina., Villar-García, María., y Maldonado-Reyes, Ana. (2017). Escenario actual del diseño gráfico en el ámbito de la investigación en México. Revista Legado de Arquitectura y Diseño, 12(21), 48-55. Recuperado de https://legadodearquitecturaydiseno.uaemex.mx/article/view/9250

Rivera Díaz, Luis Antonio. (2018). La evaluación de la educación del diseño en México: un enfoque desde la didáctica. Ciudad de México, México: Comaprod.

Rodríguez Mendoza, Ramón. (2016). La pedagogía del diseño gráfico basada en la investigación en diseño. Revisión bibliográfica. Iconofacto, 12(19), 254-267. Recuperado de https://dialnet.unirioja.es/servlet/articulo?codigo $=6302023$

Simon, Herbert. (1996). The Sciences of the Artificial. London, England: MIT Press.

Stes, Ann., Gijbels, David., y Van Petegem, Peter. (2008). Student-focused approaches to teaching in relation to context and teacher characteristics. Higher Education, 55, 255267. doi: https://doi.org/10.1007/s10734-007-9053-9

Tiburcio-García, Carmen. (2013). La sociedad red del siglo XXI y el diseño gráfico: la Universidad Iberoamericana Puebla, su propuesta y sus implicaciones en la práctica profesional según alumnos, profesores y egresados (Tesis doctoral). Universidad Iberoamericana Puebla, México. Recuperado de https://rei.iteso.mx/bitstream/handle/11117/1174/III Carmen Tiburcio.pdf?sequence=2

Tovey, Michael. (2015). Developments in Design Pedagogy. International Conference on Engineering and Product Design Education. United Kingdom: University Of Loughborough.

Zempoalteca, Beatriz., González, Juan., Barragán, Jorge., y Guzmán, Teresa. (2018). Factores que influyen en la incorporación de las Tecnologías de la Información y la Comunicación en universidades públicas: una aproximación desde la autopercepción docente. Revista de la Educación Superior, 47(186). Recuperado de http://www.scielo.org.mx/pdf/resu/v47n186/0185-2760-resu-47-186-51.pdf 
Revista indizada en

sciplo

redalyc latindex

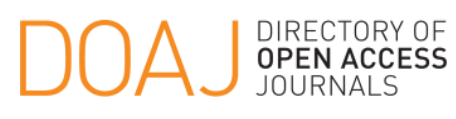

Distribuida en las bases de datos:

- Dialnet

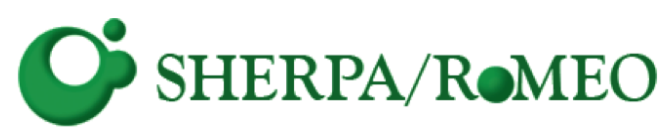

REDIB

Red Iberoamericana

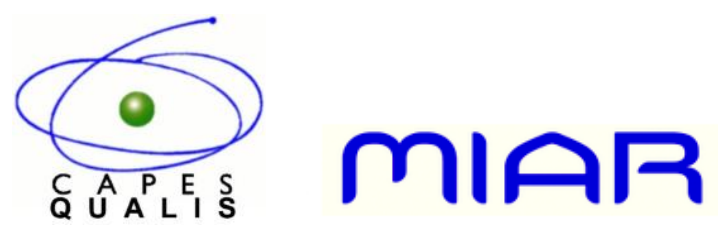

